\title{
Isolation, Biochemical Characterization and Potassium Solubilzation Efficiency of Different Microbial Isolates
}

\author{
Shreshtha Saxena ${ }^{1}$, Ravindra Kumar ${ }^{1}$, Akash Tomar ${ }^{1}$, Jitender Singh ${ }^{1}$, \\ Purushottam $^{1}$ and B. P. Dhyani ${ }^{2}$ \\ ${ }^{1}$ College of Biotechnology, ${ }^{2}$ College of Agriculture, Sardar Vallabhbhai Patel University of \\ Agriculture and Technology, Meerut, Uttar Pradesh, India-250110 \\ *Corresponding author
}

\section{A B S T R A C T}

\section{Keywords}

Potassium solubilizing bacteria (KSB), Aleksandrow media,

\section{Article Info}

Accepted:

20 May 2020

Available Online:

10 June 2020

\begin{abstract}
Microbial isolates were collected from rhizospheric soil of Meerut-Muzaffarnagar district of western Uttar Pradesh to identify the potassium solubilizing efficiency of different soil microbes. A total of thirty-eight potassium solubilizing microbes were identified and isolated on Aleksandrow Agar media. It was found that thirty-five of them were bacterial isolates and three were fungal isolates. They were further characterized based on morphological and biochemical characteristics. Among them SS 7-6, P-4-1, S-6, SS 5-3, P-1-1, SS 9-2 and SS 7-1 were Gram negative, while SS-13, SS 7-7 and P-21, were Gram positive. The highest solubilization efficiency was observed in case of SS7-6 i.e. 11.50 $\mathrm{mm}$ which was followed by SS-13 i.e. $11.30 \mathrm{~mm}$. All isolated KSB were also tested separately for their phosphorous solubilization efficiency. It was observed that the isolates namely SS 5-1, SS 5-2, SS 5-4, SS 7-1, SS7-4, S-6, SS-6, SS 9-1, SS 9-2, SS 9-3, P-1-1, PYS-1, PYS-5B, PYS-5A, P-17, P-21 and three fungal isolates efficiently solubilized phosphorous. Such 'dual-solubilizers' can convert fixed potassium and phosphorous source in soil to available form for plants.
\end{abstract}

\section{Introduction}

Potassium is one of the essential nutrient required for higher and sustainable productivity of crops. It is third important plant nutrient after Nitrogen $(\mathrm{N})$ and Phosphorus (P). Potassium (K) is required for activation of enzymes which are fundamental to metabolic processes, especially the production of proteins and sugars (Johnston, 1986). It is also essential for opening and closure of stomatal guard cells or daily changes in the orientation of leaves (Shehata and El-Khawas, 2003). $\mathrm{K}$ is present in soil with a range between $0.04-3 \%$ and only 1 to $2 \%$ of this is available to plants.

While rest of the soil bound with other minerals and is unavailable to plants (Sparks and Huang, 1985). With the progressive intensification of agriculture due to small landholdings and debut of high-yielding crop 
varieties and hybrids during green revolution, the soils are depleted in macronutrients including potassium at a speedy rate.

Moreover, available soil $\mathrm{K}$ levels have also dropped due to leaching, runoff, and erosion (Sheng and Huang, 2002a). As a after effect, potassium deficiency is becoming one of the major constraint in crop production, and therefore, many crops do respond to $\mathrm{K}$ fertilization in soils. Recently, K deficiency has also been recited in most of the crop plants (Meena, et al., 2014; Xiao, et al., 2017).

To overcome this quandary and to secure higher plant yields, farmers are reliant on chemical sources of fertilizers (Glick, 2012). While the chemical fertilizers have helped for achieving higher plant growth and yield but they have been detrimental to soil health (Adesemoye and Kloepper, 2009). Such chemical fertilizers are applied at higher than recommended doses to enhance crop yields, which have created environmental pollution problems (Brady 1990; Akande, et al., 2008).

Therefore, direct application of rock phosphate and rock potassium materials in soils may be agronomically more useful and environmentally safer than application of soluble $\mathrm{P}$ and $\mathrm{K}$ applied as chemical fertilizers. However, application of complex minerals requires presence or incorporation of their solubilizers as well (Rajan, et al., 1996).

Thus, Eco-friendly agricultural system has emerged as an important thrust area globally for long-term soil environmental sustainability and to minimize the environmental pollution associated with extensive use of chemical fertilizers. Therefore, plant growth-promoting rhizobacteria (PGPR) along with nitrogenfixing, phosphate and potassium-solubilizing bacteria are being used as biofertilizers to conserve our existing resources and to minimize environmental pollution hazards (Vessey, 2003; Ekin, 2010; Bahadur, et al., 2014; Sindhu, et al., 2014c; Meena, et al., 2014a).

\section{Materials and Methods}

\section{Sample collection}

The samples were collected from rhizospheric soils of different crops from 7-8 inches soil surface of Meerut-Muzaffarnagar District. The samples were collected in aseptic bags and transported to lab for further processing. Each sample consisted of $200 \mathrm{~g}$ soil. The details of the place and the crops of rhizospheric soil samples are furnished in Table 1.

\section{Isolation of potassium solubilizing bacteria and fungi}

One gram of rhizosphere soil was mixed thoroughly in $10 \mathrm{ml}$ distilled water and was processed by serial dilution agar plate technique (Aneja, 2002). Suitable dilutions $\left(10^{-5}\right.$ and $\left.10^{-6}\right)$ of rhizosphere solutions were plated on Aleksandrow medium $(\mathrm{Hu}$, et al., 2006): $0.5 \%$ glucose, $0.05 \%$ magnesium sulfate heptahydrate $\left(\mathrm{MgSO}_{4} \cdot 7 \mathrm{H}_{2} \mathrm{O}\right)$, $0.0005 \%$ iron (III) chloride $\left(\mathrm{FeCl}_{3}\right), 0.01 \%$ calcium carbonate $\left(\mathrm{CaCO}_{3}\right), 0.2 \%$ calcium phosphate $\left(\mathrm{Ca}_{3}\left\{\mathrm{PO}_{4}\right\}_{2}\right)$, and $0.2 \%$ potassiumaluminum-silicate $\left(\mathrm{KAlSi}_{3} \mathrm{O}_{8}\right), \mathrm{pH}$ 7.0-7.5.

The petri-plates were incubated for 3 days at room temperature $\left(30 \pm 1^{\circ} \mathrm{C}\right)$ and the colonies exhibiting clear zones were selected as potassium solubilizers from the $10^{-5}$ dilution containing plates. Cultures from these colonies were then streaked on Nutrient Agar plates for obtaining pure culture. These pure cultures were again transferred to fresh Aleksandrow medium to confirm their solubilization efficiency. 
Estimation of potassium (K) solubilization potential of native isolates

Qualitative analysis of Potassium solubilizing activity of various isolated microbes on Aleksandrow Agar medium was calculated on the basis of solubilization zone ratio (zone diameter/colony diameter) or solubilization index (Hu, et al., 2006). Solubilization efficiency and index was evaluated according to the ratio of the total diameter (halo zone colony diameter) $/ 2$ as described by EdiPremono, et al., 1996.

Solubilization Efficiency (SE)

$=(\mathrm{D}-\mathrm{d}) / 2 \quad(>5 \mathrm{~mm})$

Solubilization zone ratio / Solubilization $\operatorname{Index}(\mathrm{SI})=\mathrm{D} / \mathrm{d}$

Where,

$\mathrm{d}=$ Diameter of Mycelium/colony+growth zone.

$\mathrm{D}=$ Diameter of zone of clearance (Mycelium/colony + Clear zone)

\section{Morphological and biochemical characteristics of KSB isolates}

For morphological characterization, all the selected isolates were examined for the colony morphology, cell shape, Gram reaction and ability to form spores according to the standard procedures (Barthalomew and Mittewer, 1950). Colony characteristics such as size, shape, texture, colour, opacity and consistency were examined. The biochemical characterizations of the isolates were carried out as per the procedures mentioned in Bergey's Manual of Systematic Bacteriology 9th Edition (1993). Sugar utilization, Methyl red test (Seeley and Vandemark, 1970), Voges-Proskauer (VP) test (Seeley and Vandemark, 1970), Nitrate reduction test, Catalase test (Blazevic and Ederer, 1975), Citrate test, Motility test, Triple sugar iron test, oxidase test, lysine decarboxylase and ornithine decarboxylase test were performed.

\section{Results and Discussion}

Screening and isolation of potassium solubilizing microbial isolates

A total of eleven soil samples were collected from various places and analyzed for presence of K-solubilizing fungal and bacterial isolates. Out of eleven soil samples screened for mixed culture on Aleksandrow agar medium, the presence of 21 potassium solubilizing bacteria and 3 potassium solubilizing fungi were observed which were isolated in pure culture. Fourteen isolates were obtained from coworker in lab working on $\mathrm{P}$ solubilizers (soil samples marked with $* / * *$ ). Hence, a total of thirty-eight microbes were tested for potassium solubilization on Aleksandrow agar medium as presented in Table 1.

Above isolates were tested for their potential to solubilize potassium and their ranking was done on basis of their solubilization efficiency (SE). Results showed that all thirty-five bacterial isolates and three fungal isolates have the potential to solubilize potassium although with differing solubilization efficiency (SE value). Isolates named SS 7-6, SS-13, P-21, SS 7-7, P-1-1, S-6, SS 5-3, P-41, SS 9-2 and SS 7-1 solubilized potassium with higher solubilization efficiency. The results observed are presented in Table 2 and Fig 1. The maximum clear zone measured on Aleksandrow agar medium was $29 \mathrm{~mm}$ for sample code SS 7-6 with solubilization efficiency (SE) of $11.50 \mathrm{~mm}$ followed by sample code SS-13 with clear zone of 28.67 $\mathrm{mm}$ and solubilization efficiency (SE) of $11.30 \mathrm{~mm}$ after 15 days of incubation at $28^{\circ} \mathrm{C}$. Isolates with solubilization efficiency of 5.0 $\mathrm{mm}$ or more are generally considered as efficient solubilizers, which was demonstrated by isolates in following order namely SS 7-6 
$(11.50 \mathrm{~mm})>\mathrm{SS} 13(11.33 \mathrm{~mm})>\mathrm{P}-21$ $(10.33 \mathrm{~mm})>\mathrm{SS}$ 7-7 $(8.67 \mathrm{~mm})>\mathrm{P}-4-1(8.33$ $\mathrm{mm})>\mathrm{S} 6(7.50 \mathrm{~mm})>\mathrm{SS} 5-3(7.50 \mathrm{~mm})>$ P-1-1 $(7.33 \mathrm{~mm})>$ SS 9-2 $(7.17 \mathrm{~mm})>$ SS 7$1(7.00 \mathrm{~mm})>\operatorname{SS}-17(5.42 \mathrm{~mm})>\mathrm{SS} 9-1$ $(5.17 \mathrm{~mm})>$ SS $9-3(5.00 \mathrm{~mm})$. It was found that more number of solubilizers were isolated from rhizosperic soils of Meerut region compared to Muzaffarnagar region. Among crop rhizospheres the efficiency and presence of solubilizers were observed in following order namely potato $>$ sugarcane $>$ turmeric > mustard > mango > lemon plant field. In similar studies (Prajapati, et al., 2012) had selected the colonies which were morphologically distinct and exhibiting zone of clearance indicating Potassium solubilization; a total of 14 bacterial isolates were isolated by them as potassium solubilizers and named as KSB1 to KSB14.

As per microscopic examination held among three isolated K-solubilizing fungi F1 and F2 were identified as Aspergillus spp. and F3 was identified as Penicillium sp. Similar study was conducted by Meena, et al., 2016 and suggested that Aspergillus spp. and Penicillium spp. were more effective solubilizers than bacteria such as Bacillus spp., Paenibacillus spp., Pseudomonas spp., etc. Nineteen out of thirty-eight isolates namely SS 5-1, SS 5-2, SS 5-4, SS 7-1, SS74, S-6, SS-6, SS 9-1, SS 9-2, SS 9-3, P-1-1, PYS-1, PYS-5B, PYS-5A, PYS-7C1, P-21, F$1, \quad$ F-2 and F-3 showed ability for solubilization of both potassium and phosphorous (Pikovskaya's agar medium, 7 days of incubation at $28^{\circ} \mathrm{C}$ ). Such dualsolubilizers may significantly enhance the availability and uptake of $\mathrm{P}$ and $\mathrm{K}$ which may ultimately reflect in higher plant growth and yield.

Based on the efficiency of $\mathrm{P}$ and $\mathrm{K}$ solubilization these PGP bacterial isolates can be further investigated for their performance to enhance growth, nutrient uptake and yield of various crops. An increase in plant root length and plant growth promotion due to inoculation of Bacillus edaphicus, a potassium solubilizing bacteria (Sheng and He, 2006), maize (Wu, et al., 2005), brinjal (Ramarethinam and Chandra, 2005), cotton and rape seed (Sheng, 2005) were previously reported. K-solubilizers, apart from solubilization of potassic minerals, are also known to possess other beneficial properties like solubilization of insoluble inorganic phosphates, polysaccharide production and production of plant growth promoting substances etc. Similar, observation for solubilization of insoluble phosphates by potassium solubilizing bacteria Bacillus muciligenosus was reported earlier by $(\mathrm{Hu}$, et al., 2006). Similarly, bacteria Bacillus mucilaginosus was isolated and characterized for their ability to solubilize two potassium bearing minerals like feldspar and illite. Their probable mechanism of action for solubilization of potassium bearing minerals was predicted and shown be to the action of organic acids like oxalic acid and capsular polysaccharides (Fang and Yan, 2006). The results in the present report are in agreement with the findings of (Norkina and Pumpynaskaya, 1956), they isolated two strains of Bacillus sp. and Pseudomonas from rhizosphere soil of various crop plants as mineral potassium solubilizers.

The variability among the bacterial solubilizing ability indicates that there is a need of time for exploration of different mineral potassium solubilizing bacteria and to understand their solubilizing mechanisms. Similar study was carried out by (Anjanadevi, et al., 2015) with 36 different bacteria isolated from rocks of a major hill station at Ponmudi in Thiruvananthapuram, Kerala, India. A comprehensive characterization of $\mathrm{K}$ solubilization from feldspar was achieved with these isolates which indicated that the $\mathrm{K}$ 
solubilizing efficiency increases with decrease in $\mathrm{pH}$ and increase in viscosity and viable cell count. When mica was replaced with $\mathrm{KCl}$ and $\mathrm{K}_{2} \mathrm{SO}_{4}$ it was found that maximum $\mathrm{K}$ solubilization was obtained with $\mathrm{KCl}$ as compared to mica which in term is another aspect to study the behavior of bacteria with different substrate (Liu, 2006).

\section{Colony morphology and microscopic analysis of pure culture of different KSB isolates}

After 24 hours of incubation of these isolates on nutrient agar media colony morphology and microscopic appearance were observed as shown in Table 3. The results showed that isolates consist of twenty-seven Gram positive rod, round shaped bacteria and eight Gram negative round shaped bacteria. Similarly, (Begum, et al., 2017) collected soil samples from fish and vegetable waste dumping area of Dhaka city, Bangladesh, A total of $19 \mathrm{KSB}$ single colonies were picked up and cultured. In order to characterize bacterial isolates, gram staining and biochemical tests were performed, it was found that $79 \%$ isolates were gram positive while $26 \%$ were gram negative. In microscopic analysis, some bacteria were of round shape and some were rod shape. Also (Prajapati, et al., 2012) found that out of five selected KSB strains three were gram negative while two were gram positive bacteria. All were motile and four of them had capsule. The results are in agreement with the findings of (Norkina and Pumpynaskaya, 1956), they isolated two strains of Bacillus sp. and Pseudomonas from rhizosphere soil of various crop plants as mineral potassium solubilizers.

\section{Biochemical analysis of different isolates}

From the thirty-five bacterial isolates top ten efficient bacterial isolates were selected for studying their biochemical characteristics. Different biochemical tests (Triple sugar iron agar test, Citrate utilization test, Methyl Red Test, Voges-Proskauer test, Catalase test, Oxidase test, Motility tests, Lysine Decarboxylase Test, Ornithine decarboxylase test and Potassium Hydroxide test) were performed for these $\mathrm{K}$ solubilizing isolates. The results of different biochemical tests are summarized in Table 4.

All these ten isolates showed positive results for Citrate, Motility and Oxidase tests. For citrate utilization test appearance of dark blue color on Simmon's citrate slant from forest green color indicates positive result and confirms that citrate has been utilized. For motility test diffused growth along the stabbed line showed that bacteria were motile and indicated positive result.

All isolates showed diffused growth and hence are motile in nature. For oxidase test formation of blue color when culture was mixed with $1 \%$ solution of tetramethyl-pphenylene-diaminedihydrochloride indicates positive result. All isolates showed positive result with appearance of dark blue color.

Other biochemical tests showed mixed results for these 10 isolates. For VP test positive result was indicated by appearance of red color. Out of 10 isolates, sample code namely P-21, SS7-7, P-4-1, P-1-1, SS 7-1 and SS-13 showed positive result. Sample code SS 7-6, S6, SS 5-3, and SS 9-2 showed negative results. For catalase test formation of bubbles confirms that test was positive.

Out of 30 isolates, P-17 (PYS-7C1) and S-6 showed negative result while rest all 28 showed positive results. The Triple sugar iron agar (TSI) test was conducted on 28 isolates; it is named for its ability to test microorganism's ability to ferment sugars and to produce hydrogen sulphide. 
Table.1 Details of Potassium solubilizing microbes isolated from different rhizosperic soil samples

\begin{tabular}{|c|c|c|c|c|c|c|}
\hline S.No. & $\begin{array}{c}\text { Soil } \\
\text { Sample }\end{array}$ & Place & Date & Crop field & $\begin{array}{l}\text { Culture } \\
\text { Medium }\end{array}$ & $\begin{array}{l}\text { Isolate } \\
\text { code }\end{array}$ \\
\hline 1 & S 1 & $\begin{array}{l}\text { Location } 2 \\
\text { HRC, SVPUAT, } \\
\text { Meerut }\end{array}$ & $\begin{array}{l}09 / 02 / 2 \\
019\end{array}$ & $\begin{array}{l}\text { Turmeric } \\
\text { (Curcuma longa) }\end{array}$ & NA-KB-AM & No K- sol. \\
\hline 2 & S 2 & $\begin{array}{l}\text { Phase } 2 \text { Pallavpuram, } \\
\text { Meerut }\end{array}$ & $\begin{array}{l}12 / 02 / 2 \\
019\end{array}$ & $\begin{array}{l}\text { Onion } \\
\text { (Allium cepa) }\end{array}$ & NA-KB-AM & No K- sol. \\
\hline 3 & S 3 & $\begin{array}{l}\text { Sahaoli village, } \\
\text { Muzaffarnagar, } \\
\text { Uttar Pradesh }\end{array}$ & $\begin{array}{l}12 / 02 / 2 \\
019\end{array}$ & $\begin{array}{l}\text { Sugarcane } \\
\text { (Saccharumofficinarum) }\end{array}$ & $\mathrm{AM}$ & No K- sol. \\
\hline 4 & S 4 & $\begin{array}{l}\text { Makhyali village } \\
\text { Muzaffarnagar, } \\
\text { Uttar Pradesh }\end{array}$ & $\begin{array}{l}15 / 02 / 2 \\
019\end{array}$ & $\begin{array}{l}\text { Oat } \\
\text { (Avena sativa) }\end{array}$ & $\mathrm{AM}$ & No K- sol. \\
\hline \multirow[t]{4}{*}{5} & \multirow[t]{4}{*}{ S 5} & \multirow{4}{*}{$\begin{array}{l}\text { Mataur Village, } \\
\text { Daurala Block, Meerut } \\
\text { District, } \\
\text { Uttar Pradesh }\end{array}$} & \multirow{4}{*}{$\begin{array}{l}18 / 02 / 2 \\
019\end{array}$} & \multirow{4}{*}{$\begin{array}{l}\text { Yellow mustard } \\
\text { (B. hirta/ Sinapis alba) }\end{array}$} & \multirow[t]{4}{*}{ AM-NA } & SS 5-1 \\
\hline & & & & & & SS 5-2 \\
\hline & & & & & & SS 5-3 \\
\hline & & & & & & SS 5-4 \\
\hline 6 & S 6 & $\begin{array}{l}\text { ICAR-IIFSR Meerut } \\
\text { (UP) }\end{array}$ & $\begin{array}{l}18 / 02 / 2 \\
019\end{array}$ & $\begin{array}{l}\text { Sugarcane(spring) +black } \\
\text { gram+wheat }\end{array}$ & AM-NA/KB & SS 6 \\
\hline \multirow[t]{10}{*}{7} & \multirow[t]{10}{*}{ S 7} & \multirow{10}{*}{$\begin{array}{l}\text { ICAR-IIFSR Meerut } \\
\text { (UP) }\end{array}$} & \multirow{10}{*}{$\begin{array}{l}18 / 02 / 2 \\
019\end{array}$} & \multirow{10}{*}{$\begin{array}{l}\text { Potato } \\
\text { (Solanumtuberosum) }\end{array}$} & \multirow[t]{8}{*}{ AM-NA/KB } & SS 7-1 \\
\hline & & & & & & SS 7-2 \\
\hline & & & & & & SS 7-3 \\
\hline & & & & & & SS 7-4 \\
\hline & & & & & & SS 7-5 \\
\hline & & & & & & SS 7-6 \\
\hline & & & & & & SS 7-7 \\
\hline & & & & & & SS 7-8 \\
\hline & & & & & AM-PDA & SS F-1 \\
\hline & & & & & AM-PDA & SS F-3 \\
\hline \multirow[t]{2}{*}{8} & \multirow[t]{2}{*}{ S 8} & \multirow{2}{*}{$\begin{array}{l}\text { Siwaya-jamalullapur } \\
\text { Village, Daurala, } \\
\text { Meerut }\end{array}$} & \multirow{2}{*}{$\begin{array}{l}18 / 02 / 2 \\
019\end{array}$} & \multirow{2}{*}{$\begin{array}{l}\text { Sugarcane } \\
\text { (Saccharumofficinarum) }\end{array}$} & \multirow[t]{2}{*}{ AM-NA } & SS 8-1 \\
\hline & & & & & & SS 8-2 \\
\hline 9 & S 9 & $\begin{array}{l}\text { Daurala Block, } \\
\text { Meerut District, Uttar } \\
\text { Pradesh }\end{array}$ & $\begin{array}{l}18 / 02 / 2 \\
019\end{array}$ & $\begin{array}{l}\text { Sugarcane } \\
\text { (Saccharumofficinarum) }\end{array}$ & AM-NA/KB & $\begin{array}{l}\text { SS 9-1 } \\
\text { SS 9-2 } \\
\text { SS 9-3 }\end{array}$ \\
\hline 10 & S 10 & $\begin{array}{l}\text { A2Z Colony Green } \\
\text { Estate, Muzaffarnagar }\end{array}$ & $\begin{array}{l}18 / 02 / 2 \\
019\end{array}$ & Garden soil & AM-PDA & SS F2 \\
\hline 11 & S 11 & $\begin{array}{l}\text { Bahrala Village, } \\
\text { Daurala Block, } \\
\text { Meerut District, U.P }\end{array}$ & $\begin{array}{l}18 / 02 / 2 \\
019\end{array}$ & $\begin{array}{l}\text { Sugarcane } \\
\text { (Saccharumofficinarum) }\end{array}$ & AM-NA/KB & No K- sol. \\
\hline $12 *$ & S 12 & $\begin{array}{l}\text { Sample } 6(\mathrm{PY}) \\
\text { Vill. Shawali, MZN, } \\
\text { UP }\end{array}$ & $\begin{array}{l}27 / 02 / 1 \\
9\end{array}$ & Fruit Field & KB-AM & SS-12 \\
\hline
\end{tabular}




\begin{tabular}{|c|c|c|c|c|c|c|}
\hline $13^{*}$ & S 13 & $\begin{array}{l}\text { Sample } 3 \\
\text { Horticulture Research } \\
\text { Center, } \\
\text { SVPUAT, Meerut }\end{array}$ & $\begin{array}{l}27 / 02 / 1 \\
9\end{array}$ & $\begin{array}{l}\text { Mango } \\
\text { (Mangifera indica L.) }\end{array}$ & KB-AM & $\begin{array}{l}\text { SS } 13-1 \\
\text { SS } 13-2\end{array}$ \\
\hline $14 *$ & S 14 & $\begin{array}{l}\text { Horticulture Research } \\
\text { Center, } \\
\text { SVPUAT, Meerut }\end{array}$ & $\begin{array}{l}27 / 02 / 1 \\
9\end{array}$ & $\begin{array}{l}\text { Lemon } \\
\text { (Citrus limon) }\end{array}$ & KB-AM & P-19 \\
\hline $15^{*}$ & S 15 & $\begin{array}{l}\text { Sample - } 11 \\
\text { Horticulture Research } \\
\text { Center, } \\
\text { SVPUAT, Meerut }\end{array}$ & $\begin{array}{l}27 / 02 / 1 \\
9\end{array}$ & $\begin{array}{l}\text { Turmeric } \\
\text { (Curcuma longa) }\end{array}$ & NA/KB-AM & $\begin{array}{l}\text { SS } 15-1 \\
\text { P-3-2 }\end{array}$ \\
\hline $16^{*}$ & S 16 & $\begin{array}{l}\text { Sample }-11 \\
\text { Horticulture Research } \\
\text { Center, SVPUAT, } \\
\text { Meerut }\end{array}$ & $\begin{array}{l}27 / 02 / 1 \\
9\end{array}$ & $\begin{array}{l}\text { Turmeric } \\
\text { (Curcuma longa) }\end{array}$ & NA/KB-AM & $\begin{array}{l}\text { P 4-1 } \\
\text { SS 16-2 }\end{array}$ \\
\hline $17 *$ & S 17 & $\begin{array}{l}\text { Sample }-11 \\
\text { Horticulture Research } \\
\text { Center, SVPUAT, } \\
\text { Meerut }\end{array}$ & $\begin{array}{l}27 / 02 / 1 \\
9\end{array}$ & $\begin{array}{l}\text { Turmeric } \\
\text { (Curcuma longa) }\end{array}$ & NA/KB-AM & SS-17 \\
\hline $18^{*}$ & S 18 & $\mathrm{HRC}$ & $\begin{array}{l}27 / 02 / 1 \\
9\end{array}$ & Uncultivated & NA/KB-AM & SS-18 \\
\hline $19 *$ & S 19 & $\mathrm{HRC}$ & $\begin{array}{l}27 / 02 / 1 \\
9\end{array}$ & - & NA/KB-AM & SS-19 \\
\hline $20 *$ & S 20 & HRC & $\begin{array}{l}27 / 02 / 1 \\
9\end{array}$ & $\begin{array}{l}\text { Turmeric } \\
\text { (Curcuma longa) }\end{array}$ & NA/KB-AM & SS-20 \\
\hline $21 * *$ & S 21 & HRC & $\begin{array}{l}27 / 02 / 1 \\
9\end{array}$ & - & KB-AM & P-1-1 \\
\hline $22 * *$ & S 22 & $\begin{array}{l}\text { Sample } 1 \text { (PY) } \\
\text { Horticulture Research } \\
\text { Center, } \\
\text { SVPUAT, Meerut }\end{array}$ & $\begin{array}{l}27 / 02 / 1 \\
9\end{array}$ & $\begin{array}{l}\text { Turmeric } \\
\text { (Curcuma longa) }\end{array}$ & KB-AM & PYS-1 \\
\hline $23 * *$ & S 23 & $\begin{array}{l}\text { Sample } 5 \text { (PY) } \\
\text { MZN, UP }\end{array}$ & $\begin{array}{l}27 / 02 / 1 \\
9\end{array}$ & $\begin{array}{l}\text { Sugarcane } \\
\text { (Saccharum officinarum) }\end{array}$ & KB-AM & $\begin{array}{l}\text { PYS-5A } \\
\text { PYS-5B }\end{array}$ \\
\hline $24 * *$ & S 24 & $\begin{array}{l}\text { Sample } 7(\mathrm{PY}) \\
\text { Almaspur, } \\
\text { Muzaffarnagar }\end{array}$ & $\begin{array}{l}27 / 02 / 1 \\
9\end{array}$ & $\begin{array}{l}\text { Water chestnut } \\
\text { (Trapa natans) }\end{array}$ & KB-AM & P-17 \\
\hline $25 * *$ & S 25 & $\begin{array}{l}\text { Hill region, } \\
\text { Uttrakhand. }\end{array}$ & $\begin{array}{l}27 / 02 / 1 \\
9\end{array}$ & $\begin{array}{l}\text { Department of Soil } \\
\text { Science, COA, } \\
\text { SVPUAT, Meerut }\end{array}$ & KB-AM & $\mathrm{P}-21$ \\
\hline $\begin{array}{l}\# \# \\
* \\
* *\end{array}$ & $\begin{array}{l}\text { AM } \\
\text { B M } \\
\text { Isoli } \\
\text { Isoli } \\
\text { Isol: }\end{array}$ & $\begin{array}{l}\text { ksandrow Medium, PDA- } \\
\text { m. KS- potassium solubili } \\
\text { rom S.no } 12 \text { to S. no } 25 \\
\text { bove were not solubilizing } \\
\text { bove were solubilizing ph }\end{array}$ & $\begin{array}{l}\text { tato Dext } \\
\text { e taken fro } \\
\text { hosphorus } \\
\text { phorus an }\end{array}$ & $\begin{array}{l}\text { ose Agar, KB-Kings } \\
\text { n co-worker in lab. } \\
\text { but solubilized potassium. } \\
\text { potassium both. }\end{array}$ & & \\
\hline
\end{tabular}


Table.2 Potassium solubilizing efficiency of different microbial isolates

\begin{tabular}{|c|c|c|c|c|c|c|c|c|}
\hline \multirow[t]{2}{*}{ S. } & \multirow[t]{2}{*}{$\begin{array}{l}\text { Isolates } \\
\text { Codes }\end{array}$} & \multicolumn{2}{|c|}{$\begin{array}{l}\text { Colony Size } \\
\text { (d) }(\mathbf{m m})\end{array}$} & \multicolumn{2}{|c|}{$\begin{array}{l}\text { Solubilization Zone } \\
\text { (D) (mm) }\end{array}$} & \multirow{2}{*}{$\begin{array}{c}\begin{array}{c}\text { Solubilization } \\
\text { Efficiency } \\
(\mathbf{m m})\end{array} \\
(\mathrm{D}-\mathbf{d}) / 2\end{array}$} & \multirow[t]{2}{*}{ Rank } & \multirow{2}{*}{$\begin{array}{c}\text { Phosphoru } \\
\text { S } \\
\text { solubilizin } \\
\text { g Ability }\end{array}$} \\
\hline & & d & SD & D & SD & & & \\
\hline 1 & SS 7-6 & 6.00 & \pm 0.00 & 29.00 & \pm 0.00 & 11.50 & 1 & + \\
\hline 2 & SS 13 & 6.00 & \pm 0.00 & 28.67 & \pm 0.58 & 11.33 & 2 & - \\
\hline 3 & P-21 & 9.00 & \pm 1.00 & 29.67 & \pm 0.58 & 10.33 & 3 & + \\
\hline 4 & SS 7-7 & 6.67 & \pm 2.08 & 24.00 & \pm 1.00 & 8.67 & 4 & - \\
\hline 5 & P-4-1 & 9.00 & \pm 0.00 & 25.67 & \pm 0.58 & 8.33 & 5 & - \\
\hline 6 & S 6 & 6.00 & \pm 0.00 & 21.00 & \pm 1.00 & 7.50 & 6 & + \\
\hline 7 & SS 5-3 & 6.00 & \pm 0.00 & 21.00 & \pm 1.00 & 7.50 & 7 & - \\
\hline 8 & P-1-1 & 8.33 & \pm 0.58 & 23.00 & \pm 1.00 & 7.33 & 8 & + \\
\hline 9 & SS 9-2 & 5.33 & \pm 0.58 & 19.67 & \pm 2.08 & 7.17 & 9 & + \\
\hline 10 & SS 7-1 & 6.00 & \pm 0.00 & 20.00 & \pm 0.00 & 7.00 & 10 & + \\
\hline 11 & SS-17 & 7.33 & \pm 0.58 & 18.17 & \pm 0.29 & 5.42 & 11 & - \\
\hline 12 & SS 9-1 & 6.33 & \pm 1.15 & 16.67 & \pm 1.53 & 5.17 & 12 & + \\
\hline 13 & SS 9-3 & 5.67 & \pm 0.58 & 15.67 & \pm 4.04 & 5.00 & 13 & + \\
\hline 14 & PYS-1 & 7.33 & \pm 1.15 & 16.33 & \pm 0.58 & 4.50 & 14 & + \\
\hline 15 & SS 7-5 & 7.00 & \pm 2.00 & 16.00 & \pm 0.00 & 4.50 & 15 & - \\
\hline 16 & SS $7-3$ & 7.00 & \pm 1.73 & 16.00 & \pm 0.00 & 4.50 & 16 & - \\
\hline 17 & SS 5-1 & 7.67 & \pm 0.58 & 15.67 & \pm 0.58 & 4.00 & 17 & + \\
\hline 18 & SS 7-4 & 6.33 & \pm 1.15 & 13.67 & \pm 2.31 & 3.67 & 18 & + \\
\hline 19 & P-19 & 6.67 & \pm 0.58 & 13.67 & \pm 2.31 & 3.50 & 19 & - \\
\hline 20 & P-17 & 4.33 & \pm 0.58 & 11.00 & \pm 1.00 & 3.33 & 20 & + \\
\hline 21 & SS 5-2 & 8.00 & \pm 0.00 & 14.67 & \pm 0.58 & 3.33 & 21 & + \\
\hline 22 & SS 7-8 & 8.00 & \pm 0.00 & 14.33 & \pm 1.15 & 3.17 & 22 & - \\
\hline 23 & SS 15-1 & 8.67 & \pm 0.58 & 15.00 & \pm 0.00 & 3.17 & 23 & - \\
\hline 24 & SS-18 & 10.33 & \pm 0.58 & 16.00 & \pm 1.00 & 2.83 & 24 & - \\
\hline 25 & SS-6 & 7.33 & \pm 3.21 & 12.33 & \pm 2.31 & 2.50 & 25 & - \\
\hline 26 & SS-19 & 4.33 & \pm 0.58 & 8.33 & \pm 0.58 & 2.00 & 26 & - \\
\hline 27 & SS 5-4 & 8.67 & \pm 0.58 & 11.33 & \pm 0.58 & 1.33 & 27 & + \\
\hline 28 & SS 16-2 & 10.00 & \pm 1.00 & 12.00 & \pm 0.00 & 1.00 & 28 & - \\
\hline 29 & P 3-2 & 8.00 & \pm 0.00 & 10.00 & \pm 0.00 & 1.00 & 29 & - \\
\hline 30 & SS 7-2 & 5.67 & \pm 0.76 & 7.67 & \pm 0.58 & 1.00 & 30 & - \\
\hline 31 & PYS-5A & 8.33 & \pm 0.58 & 10.17 & \pm 0.76 & 0.92 & 31 & + \\
\hline 32 & SS 8-1 & 6.33 & \pm 0.29 & 8.17 & \pm 0.29 & 0.92 & 32 & - \\
\hline
\end{tabular}

* d- Colony diameter, D- diameter of potassium solubilization zone. Readings in three replica.

+: Phosphorus solubilizing Ability Present

-: Phosphorus solubilizing Ability Absent 
Table.3 Colony morphology and microscopic characteristics of top ten KSB isolates

\begin{tabular}{|c|c|c|c|c|c|c|c|c|}
\hline $\mathbf{S}$ & $\begin{array}{c}\text { Isolate } \\
\text { code }\end{array}$ & \multicolumn{2}{|c|}{ Form } & Elevation & Size & Opacity & Color & Surface \\
\hline $\mathbf{1}$ & SS 7-6 & Irregular & Flat & Small & Translucent & White & Glistering & $\begin{array}{c}\text { G-ve } \\
\text { cocci }\end{array}$ \\
\hline $\mathbf{2}$ & SS 13 & Regular & Flat & Small & Opaque & White & Dull & $\begin{array}{c}\text { G+ve } \\
\text { cocci }\end{array}$ \\
\hline $\mathbf{3}$ & P-21 & Regular & Flat & Medium & Opaque & White & Dull & $\begin{array}{c}\text { G+ve } \\
\text { small rods }\end{array}$ \\
\hline $\mathbf{4}$ & SS 7-7 & Regular & Flat & Small & Translucent & White & Glistering & $\begin{array}{c}\text { G+ve } \\
\text { cocci }\end{array}$ \\
\hline $\mathbf{5}$ & P-4-1 & Filamentous & Raised & Medium & Translucent & White & Glistering & $\begin{array}{c}\text { G-ve } \\
\text { cocci }\end{array}$ \\
\hline $\mathbf{6}$ & S-6 & Regular & Flat & Small & Transparent & White & Glistering & $\begin{array}{c}\text { G-ve } \\
\text { small rods }\end{array}$ \\
\hline $\mathbf{7}$ & SS 5-3 & Irregular & Flat & Medium & Translucent & White & Smooth & $\begin{array}{c}\text { G-ve } \\
\text { small rods }\end{array}$ \\
\hline $\mathbf{8}$ & P-1-1 & Regular & Flat & Small & Transparent & White & Rough & $\begin{array}{c}\text { G-ve } \\
\text { cocci }\end{array}$ \\
\hline $\mathbf{9}$ & SS 9-2 & Irregular & Flat & Medium & Translucent & White & Smooth & $\begin{array}{c}\text { G-ve } \\
\text { small rods }\end{array}$ \\
\hline $\mathbf{1 0}$ & SS 7-1 & Regular & Flat & Small & Translucent & White & Smooth & $\begin{array}{c}\text { G-ve } \\
\text { small rods }\end{array}$ \\
\hline
\end{tabular}

Table.4 Biochemical test of top ten KSB isolates

\begin{tabular}{|c|c|c|c|c|c|c|c|c|c|c|c|}
\hline \multirow[t]{2}{*}{ S. } & \multirow{2}{*}{$\begin{array}{c}\text { Biochemical } \\
\text { Characteristics }\end{array}$} & \multicolumn{10}{|c|}{ KSB isolates } \\
\hline & & SS 7-6 & SS-13 & P-21 & $\begin{array}{c}\text { SS 7- } \\
7\end{array}$ & $\begin{array}{c}\text { P-4- } \\
1\end{array}$ & $\begin{array}{c}S- \\
6\end{array}$ & $\begin{array}{c}\text { SS 5- } \\
3\end{array}$ & P-1-1 & $\begin{array}{c}\text { SS 9- } \\
2\end{array}$ & SS 7-1 \\
\hline 1. & Citrate utilization & + & + & + & + & + & + & + & + & + & + \\
\hline 2. & $\begin{array}{c}\text { Vogues Proskauer } \\
\text { (VP) }\end{array}$ & - & + & + & + & + & - & - & + & - & + \\
\hline 3. & Motility & + & + & + & + & + & + & + & + & + & + \\
\hline 4. & Catalase & + & + & + & + & + & - & + & + & + & + \\
\hline 5. & Oxidase & + & + & + & + & + & + & + & + & + & + \\
\hline 6. & TSI & + & + & + & + & + & - & - & - & - & + \\
\hline 7. & Methyl Red (MR) & + & - & - & - & - & - & + & - & - & + \\
\hline 8. & $\begin{array}{c}\text { Lysine } \\
\text { Decarboxylase }\end{array}$ & + & - & - & - & - & + & + & - & + & - \\
\hline 9. & $\begin{array}{c}\text { Ornithine } \\
\text { Decarboxylase }\end{array}$ & + & - & - & - & - & + & + & - & + & - \\
\hline 10. & Nitrate Reduction & - & - & - & - & + & + & - & - & + & - \\
\hline 11. & KOH Test & + & - & - & + & + & + & + & + & + & + \\
\hline
\end{tabular}




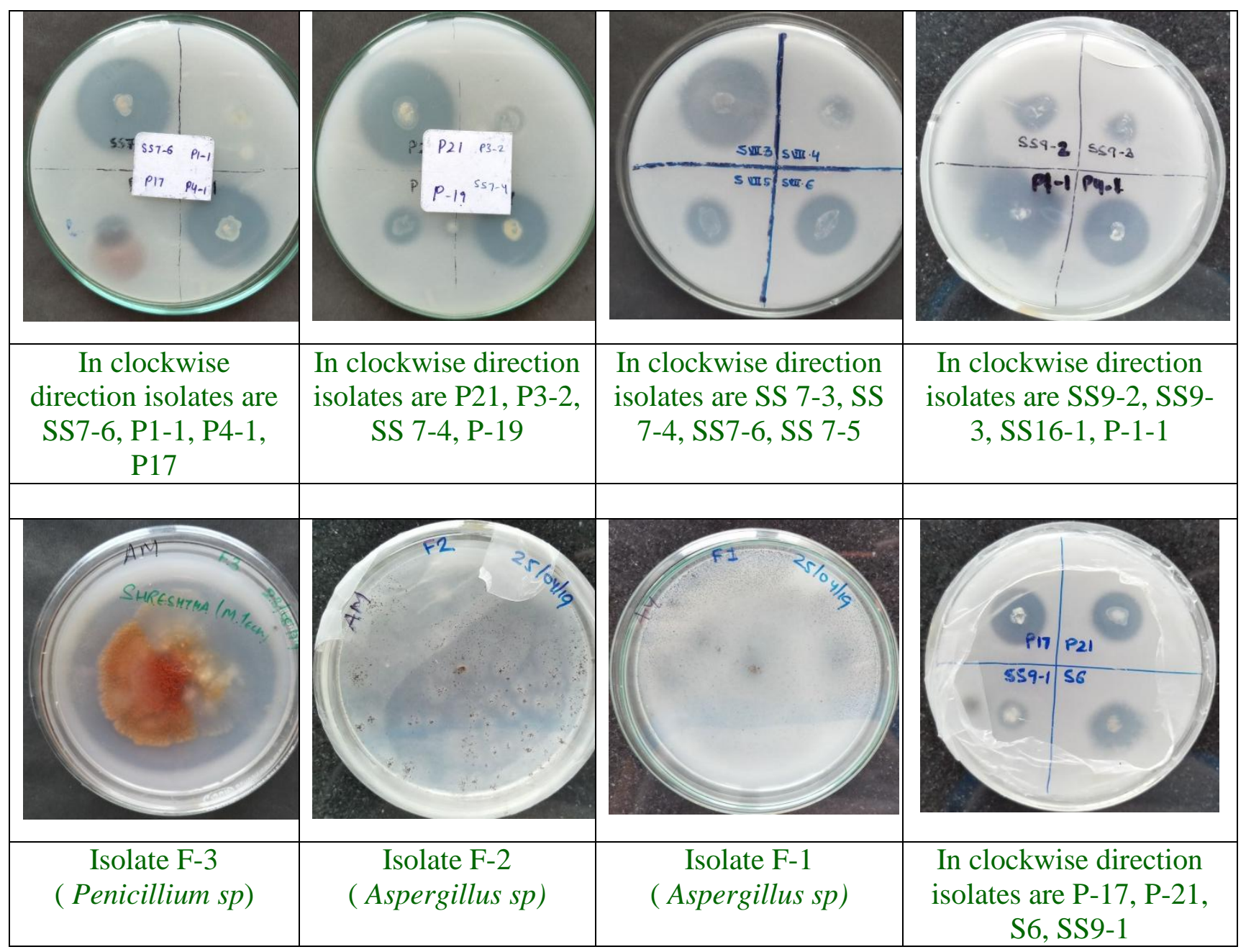

Figure.1 Potassium solubilization by different isolates on Aleksandrow agar medium

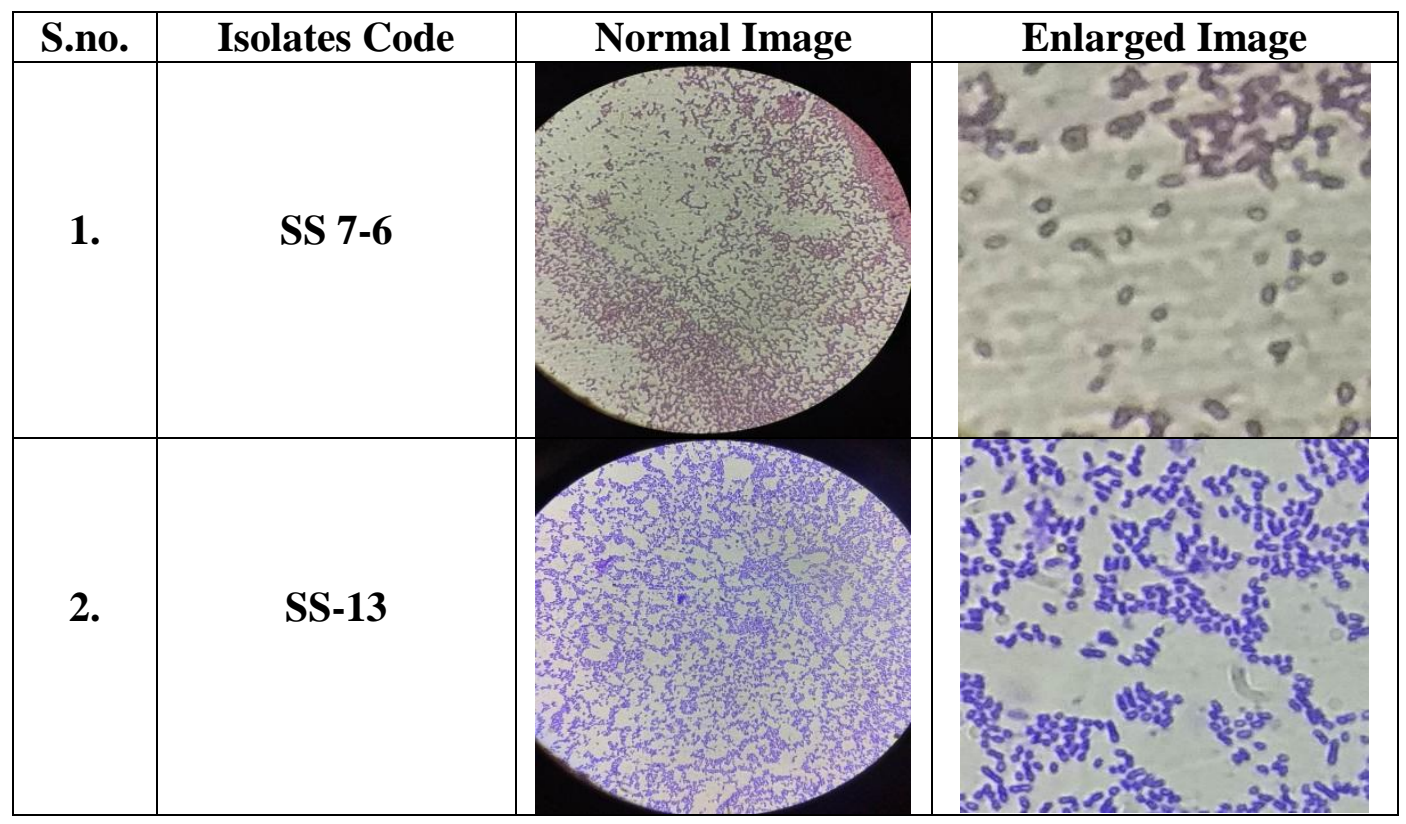




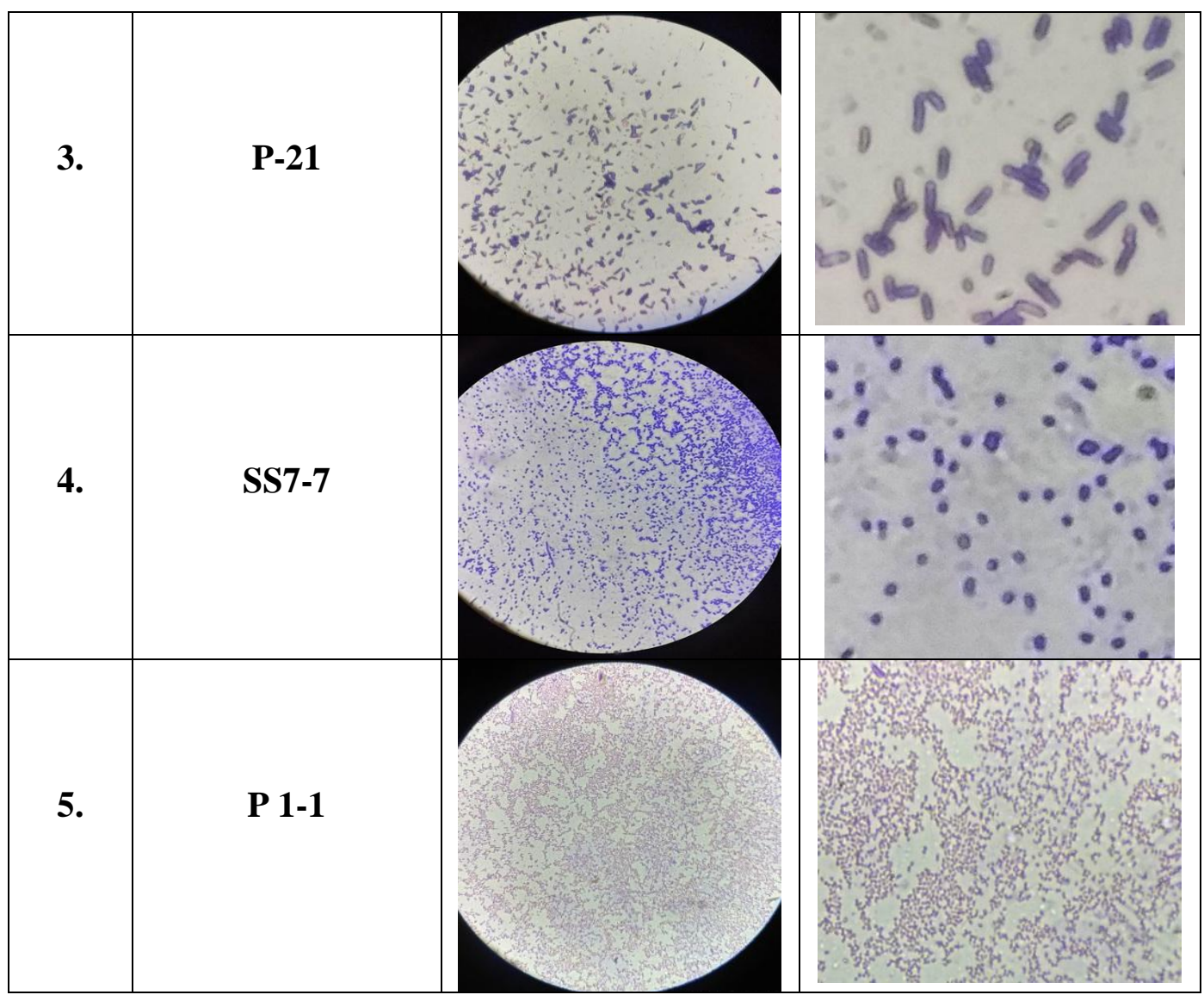

Figure.2 Microscopic characteristics and gram staining of different K solubilizing isolates

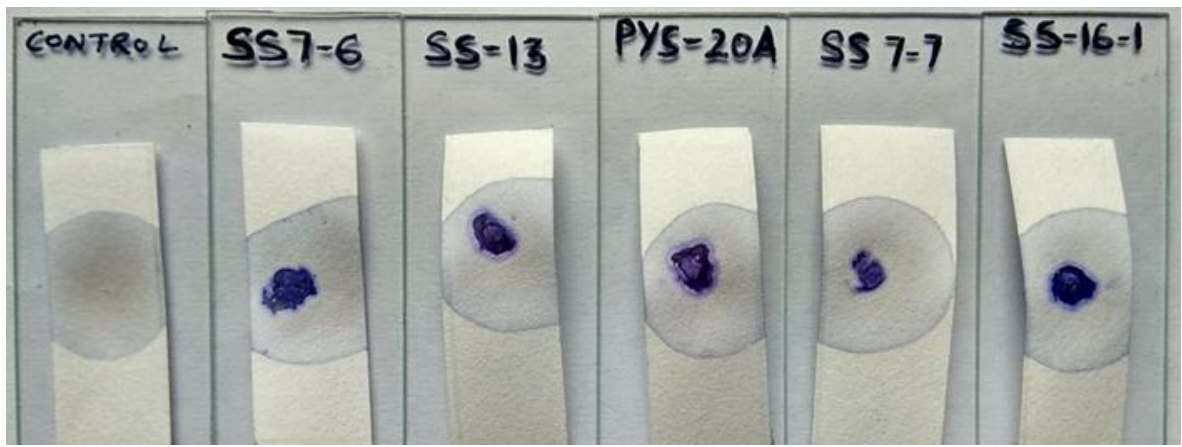

Figure.3 Oxidase test for different KSB Isolates

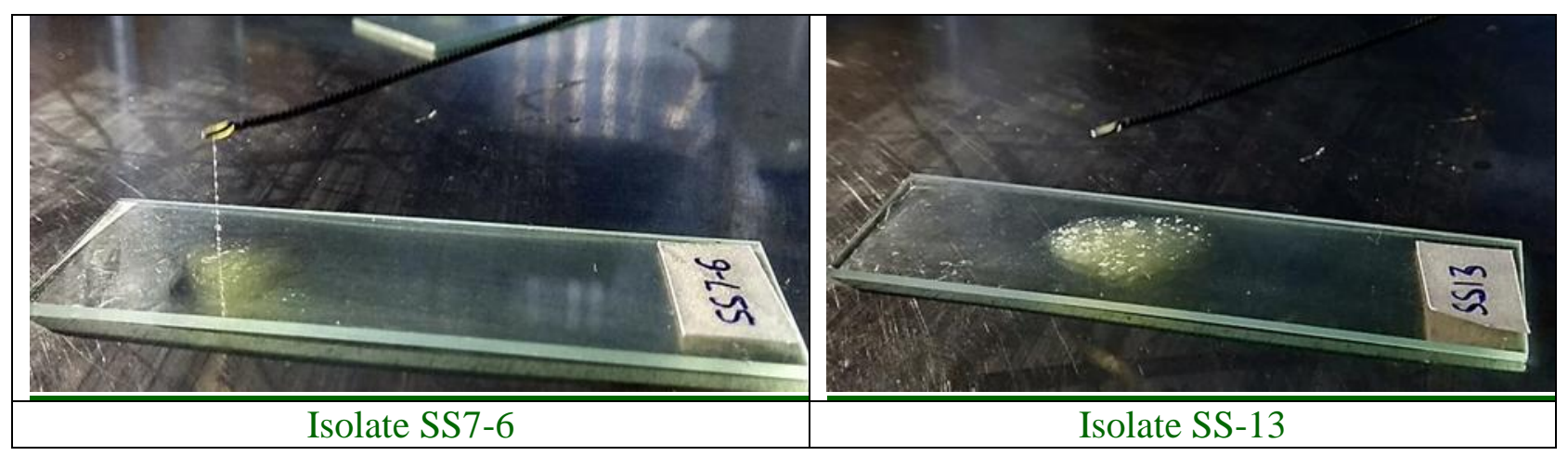




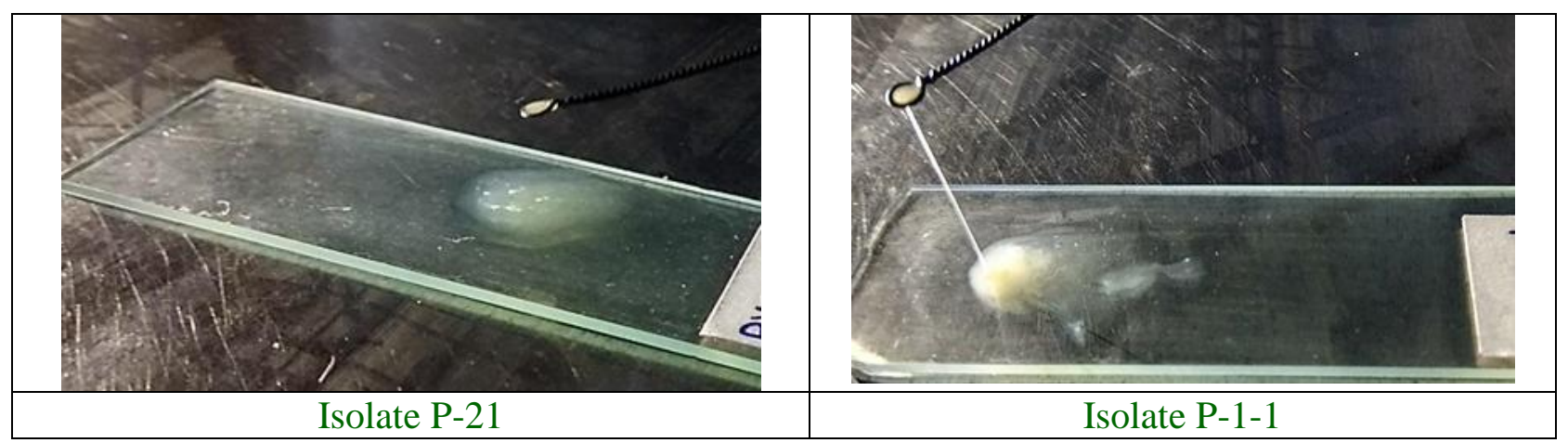

Figure.4 Potassium Hydroxide Test for KSB Isolates

It was observed that out of 28 isolates, Sample code namely SS 7-6, SS-13, PYS 20A, SS 7-7, P-4-1, SS 7-1, SS-17, SS 9-1, PYS-1, SS 7-5, SS 7-8, SS 5-1, SS 7-4, PYS7C1, SS 7-8, SS 15-1, SS-18, SS 16-2, SS 152 showed positive results. Sample code namely S-6, SS 5-3, P-1-1, SS 9-2, SS 9-3, PYS-1, SS-14, SS 5-2, SS-6, SS-19 and SS 54 were not able to ferment any sugar. It was observed that none of the isolates produced hydrogen sulphide or any of the gas $\left(\mathrm{CO}_{2} / \mathrm{H}_{2}\right)$. Sample code namely SS-13, P-21, SS 7-7, P4-1, SS -17, SS 9-1, SS 5-1, SS 7-4, PYS7C1, SS 15-1, SS-18, SS 16-2 and SS 15-2 were able to ferment only glucose because the color of the butt was observed to be yellow while slant remained red.

For methyl red test positive result was indicated by appearance of stable red color in the medium after the addition of methyl red indicator. Out of 10 isolates, sample code namely SS 7-6, SS 7-1 and SS 5-3 showed positive result. Sample code namely SS-13, P21, SS 7-7, P-4-1, SS 9-2, P-1-1 and S-6 showed negative results. For lysine decarboxylase test if the inoculated medium is yellow, or if there was no color change, the organism was decarboxylase-negative for the amino acid. If the medium turns purple, the organism was decarboxylase-positive for amino acid. Out of 10 isolates namely SS 7-6, S6, SS 5-3, SS 9-2 showed positive result and SS-13, P-21, SS 7-7, P-4-1, P-1-1, SS 7-1 showed negative result.
For ornithine decarboxylase test if the inoculated medium was yellow, or if there was no color change, the organism was decarboxylase-negative for the amino acid. If the medium turns purple, the organism was decarboxylase-positive for amino acid. Out of 10 isolates namely SS 7-6, S6, SS 5-3, SS 9-2 showed positive result and SS-13, P-21, SS 77, P-4-1, P-1-1, SS 7-1 showed negative result. For nitrate reduction test appearance of red color indicated positive result. Out of 10 isolates, sample code namely P-4-1, S-6, and SS9-2 showed light red color and sample code SS 7-6, SS-13, P-21, SS 7-7, SS 5-3, P-1-1 and SS 7-1 showed negative result.

For Potassium hydroxide test if smear of organism become thick, stringy and form long strands within the first $30 \mathrm{sec}$, it was seen as positive result for Gram negative bacteria. If organism leaves the suspension unaltered or absence of stringing, this was seen as indication for Gram positive bacteria. Eight out of 10 isolates showed positive result with long strand formation indicating gram negative bacteria and 2 isolates namely P-21 and SS 13 showed no strand formation; hence they were gram positive bacteria as shown in fig. 4.

The potassium fertilizer currently used in agriculture requires a greater input that makes them unaffordable by the farmers of developing nations. Since most soils are deficient in plant-available potassium and 
chemical fertilizers are not cost-effective, scientists thus have a responsibility for society to find ways and means of making natural potassium resources available to crops, as an economically efficient substitute for expensive chemical fertilizers. Keeping the above in view it will be of crucial importance to identify other efficient rhizosphere competent bacteria (RCB) or soil microorganisms responsible for higher potassium-solubilizing ability. In current studies K solubilizing isolates namely SS 7-6, SS-13, P-21, SS 7-7 and P-4-1 could be further tested for their use as bio inoculants for various field crops.

\section{References}

Adesemoye, A. O. and Kloepper, J. W. (2009). Plant-microbes interactions in enhanced fertilizer-use efficiency. Appl. Microbiol. Biotechnol. 85:1-12.

Akande, M. O., Adediran, J. A., Oluwatoyinbo, F. I., Makinde, E. A. and Adetunji, M. T. (2008). Suitability of poultry manure amended Sokoto rock phosphate on growth, nutrient uptake and yield of chilli pepper (Capsicum fruitscens L.). Niger. J. Soil Sci. 18:167-174.

Altamare, C., Norvell, W. A., Bjorkman, T. and Harman, G. E. (1999). Solubilization of phosphates and micronutrients by the plant growth promoting and bacterial fungus Trichodera harzianum Rifai, App. Environ. Microbiol. 65:2926-2933.

Aneja, K. R. (2002). Experiments in Microbiology, Plant Pathology, Tissue culture and Mushroom production technology, New Age International (P) Ltd., New Delhi.

Anjanadevi, P. I., John, S. N., John, S. K., Jeeva, L. M. and Misra, S. R. (2015). Rock inhabiting potassium solubilizing bacteria from Kerala, India: characterization and possibility in chemical $\mathrm{K}$ fertilizer substitution. J. Basic Microbiol. 56:67-77.

Bahadur, I., Meena, V. S. and Kumar, S. (2014). Importance and application of potassic biofertilizer in Indian agriculture. Int. Res. J.
Biol. Sci. 3:80-85.

Barthalomew, J.W. and Mittewer, J. (1950). A simplified bacterial strain. Stain Tech. 25:153.

Begum, K., Mannan, J. S., Rezwan, R., Rahman, M. Md., Rahman, S. Md. and Kamal, A. N. E. (2017). Isolation and Characterization of Bacteria with Biochemical and Pharmacological Importance from Soil Samples of Dhaka City. Dhaka Univ. J. Pharm. Sci. 16(1):129-136.

Blazevic, D. J. and Ederer, G. M. (1975). Principles of bio-chemical tests in diagnostic microbiology Wiley and Company, New York, pp.13-45.

Brady, N. C. (1990). The nature and properties of soils. Macmillan, New York, 351-380.

Edi-Premono, Moawad, M. A. and Vleck, P. L. G. (1996). Effect of phosphate solubilizing Pseudomonas putida on the growth of maize and its survival in the rhizosphere. JACS. 11:13-23.

Ekin, Z. (2010). Performance of phosphate solubilizing bacteria for improving growth and yield of sunflower (Helianthus annuus L.) in the presence of phosphorus fertilizer. Afr. J. Biotechnol. 9:3794-3800.

Fang, S. X. and Yan, L. H. (2006). Solubilization of potassium bearing minerals by wild type strain of Bacillus edaphicus and its mutants and increased potassium by wheat. Can. J. Microbiol. 52:66-72.

Glick, B. R. (2012). Plant Growth-Promoting Bacteria: Mechanisms and Applications. Scientifica. 963401.

$\mathrm{Hu}, \mathrm{X}$. F., Chen, J. and Guo, J. F. (2006). Two phosphate and potassium solubilizing bacteria isolated from Tiannu mountain, Zhejiang, China. World J. Micro.Biotech. 22: 983-990.

Johnston, A. E. (1986). Potassium fertilization to maintain imbalance under various farming system. In: Nutrient Balance and the Need for Potassium. Proceedings of 13th Congress of the International Potash Institute. International Potash Institute, Basel, Switzerland, pp.199-226.

Liu, W., Xu, X., Wu, S., Yang, Q., Luo, Y. and Christie, P. (2006). Decomposition of silicate minerals by Bacillus mucilaginosus in liquid culture. Environ. Geochem. Health, 
28:133-140.

Meena, V. S., Bahadur, I., Maurya, B. R., Kumar, A., Meena, R. K., Meena, S. K., Verma, J. P. (2016).

Potassium-Solubilizing Microorganism in Evergreen Agriculture: An Overview. Springer.

Meena, V. S., Maurya, B. R. and Verma, J. P. (2014b). Does a rhizospheric microorganism enhance $\mathrm{K}^{+}$availability in agricultural soils? Microbiol. Res. 169:337-347.

Norkina, S. P. and Pumpyansakya, L. V. (1956). Certain properties of silicate bacteria dokl. Crop Sci., Soc. Japan, 28:35-40.

Prajapati, B. K. and Modi, A. H. (2012). Isolation and characterization of potassium solubilizing bacteria from ceramic industry soil. CIB Tech. Journal of Microbiology, Vol. 1 (2-3) Jul.-Sept. \& Oct.-Dec., pp.8-14.

Rajan, S. S. S., Watkinson, J. H. and Sinclair, A. G. (1996). Phosphate rock for direct application to soils. Adv. Agron. 57:77-159.

Ramarethinam, S. and Chandra, K. (2005). Studies on the effect of potash solubilizing/mobilizing bacteria Frateuria aurantia on Brinjal growth and yield. Pestol. 11:35-39.

Seeley, H.W. and Vandemark, P. J. (1970). Microbes in action: A laboratory manual of microbiology, D. P. TarapoRevale Sons and Company Ltd., Bombay, pp.86-95.

Shehata, M. M. and El-khawas, S. A. (2003). Effect of two biofertilizers on growth parameters, yield characters, nitrogenous components, nucleic acid contents, minerals, oil content, protein profiles and DNA banding pattern of sunflower (Helianthus annus L. Cv. Vedock) yield. Pak. J. Biol. Sci. 6(14):1257-1268.

Sheng, X. F. (2005). Growth promotion and increased potassium uptake of cotton and rape by a potassium releasing strain of Bacillus edaphicus. Soil Bio. Biochem. 37(1):1918-1922.

Sheng, X. F. and He, L. Y. (2006). Solubilization of potassium-bearing minerals by a wildtype strain of Bacillus edaphicus and its mutants and increased potassium uptake by wheat. Can. J. Microbiol. 52(1):66-72.

Sheng, X. F. and Huang, W. Y. (2002a). Study on the conditions of potassium release by strain NBT of silicate bacteria. Sci. Agric. Sin. 35:673-677.

Sindhu, S. S, Phour, M., Choudhary, S. R. and Chaudhary, D. (2014c). Phosphorus cycling: prospects of using rhizosphere microorganisms for improving phosphorus nutrition of plants. In: Parmar N, Singh A (eds) Geomicrobiology and biogeochemistry. Berlin/Heidelberg, 199-237.

Sparks and Huang, P. M. (1985). Physical chemistry of soil potassium. In Potassium in agriculture (ed.) Munson, R. D., American Soc. Agron. J. 201-276.

Vessey, K. J. (2003). Plant growth promoting rhizobacteria as biofertilizers. Plant Soil, 25:557-586.

Wu, S. C., Cao, Z. H., Li, Z. G., Cheung, K. C. and Wong, M. H. (2005). Effect of biofertilizer containing $\mathrm{N}$-fixer, $\mathrm{P}$ and $\mathrm{K}$ solubilizers and AM-fungi on maize growth. Geoderma, 125:155-166.

Xiao, Y., Wang, X., Chen, W. and Huang, Q. (2017). Isolation and identification of three potassium-solubilizing bacteria from rape rhizospheric soil and their effects on ryegrass. Geomicrobiol. J. 1-8.

\section{How to cite this article:}

Shreshtha Saxena, Ravindra Kumar, Akash Tomar, Jitender Singh, Purushottam and Dhyani. B. P. 2020. Isolation, Biochemical Characterization and Potassium Solubilzation Efficiency of Different Microbial Isolates. Int.J.Curr.Microbiol.App.Sci. 9(06): 2667-2680. doi: https://doi.org/10.20546/ijcmas.2020.906.325 